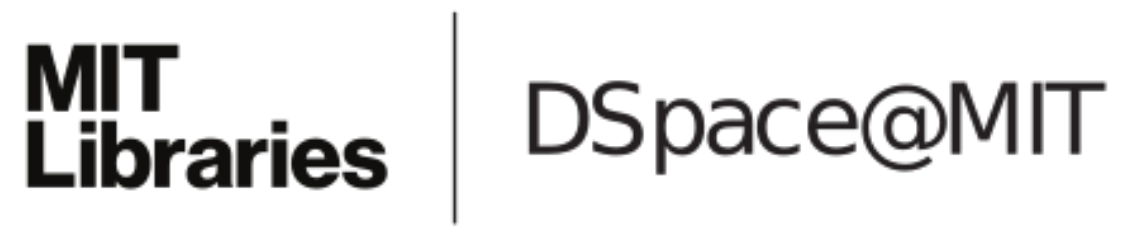

\author{
MIT Open Access Articles
}

Depth resolved wide field illumination for biomedical imaging and fabrication

The MIT Faculty has made this article openly available. Please share how this access benefits you. Your story matters.

Citation: Siemens, M. et al. “EUV detection of high-frequency surface acoustic waves." Lasers and Electro-Optics, 2009 and 2009 Conference on Quantum electronics and Laser Science Conference. CLEO/QELS 2009. Conference on. 2009. 1-2. (c) 2009 IEEE.

As Published: http://dx.doi.org/10.1109/IEMBS.2009.5333601

Publisher: Institute of Electrical and Electronics Engineers

Persistent URL: http://hdl.handle.net/1721.1/61658

Version: Author's final manuscript: final author's manuscript post peer review, without publisher's formatting or copy editing

Terms of Use: Article is made available in accordance with the publisher's policy and may be subject to US copyright law. Please refer to the publisher's site for terms of use. 


\title{
Depth Resolved Wide Field Illumination for Biomedical Imaging and Fabrication
}

\author{
Peter T. C. So and Daekeun Kim
}

\begin{abstract}
Nonlinear microscopic imaging is relatively slow due to the sequential nature of raster scanning. Recently, this limitation was overcome by developing a 3D-resolved wide-field two-photon microscope based on the concept of temporal focusing. The existing temporal focusing systems have poor optical sectioning capability and, due to a shortage of illumination power, low actual frame rate. In this presentation, a comprehensive mathematical model for temporal focusing two-photon microscope will be presented. By optimizing instrument design and the use of high two-photon cross section quantum dots, we demonstrate single quantum dot imaging with submicron resolution at video rate and applied it to study transport processes in cells. Further, we realize that the depth resolved wide field illumination can be used for microfabrication. A prototype three-dimensional lithographic microfabrication system was developed and micropatterning capability based on photobleaching process is demonstrated.
\end{abstract}

\section{INTRODUCTION}

$\mathrm{N}$ ONLINEAR excitation techniques is the method of choice for deep tissue microscopic imaging and 3D microfabrication. Nonlinear imaging is now used routinely in neurobiology, immunology and holds potential for non-invasive biopsy of cancer. The 3D resolved nature of nonlinear excitation has been further used for microfabrication and microprocessing. Preliminary experiments to build photonic crystals, tissue engineering constructs, and MEMS devices have been demonstrated based on 3D processing and fabrication using nonlinear excitation.

While nonlinear excitation is a powerful technique for 3D imaging and fabrication, imaging speed and fabrication rate based on this technique is slow. Nonlinear excitation typically achieves $3 \mathrm{D}$ resolution based on spatial focusing of infrared light at the focal point of a high numerical aperture lens. The nonlinear dependence of excitation efficiency on the input light intensity distribution results in a 3D confined point spread function (PSF) that is femtoliter in volume. While this approach has high resolution, its imaging and fabrication speed is limited by the need to sequentially scan this excitation volume in 3D. Recently, two research groups $[1,2]$ have developed a new depth resolved wide field illumination (DRWFI) technique based on temporal

Manuscript received June 3, 2009. This work was supported in part by Singapore-MIT Alliance 2 and Singapore-MIT Alliance for Science and Technology Center.

P. T. C. So is with the Department of Mechanical Engineering and the Department of Biological Engineering in the Massachusetts Institute of Technology, Cambridge, MA 02139USA (phone: 617-253-6552; e-mail: ptso@MIT.EDU).

D. Kim is is with the Department of Mechanical Engineering in the Massachusetts Institute of Technology, Cambridge, MA 02139USA focusing. The basic idea of DRWFI based on temporal focusing and its comparison with spatial focusing is represented in Fig. 1.

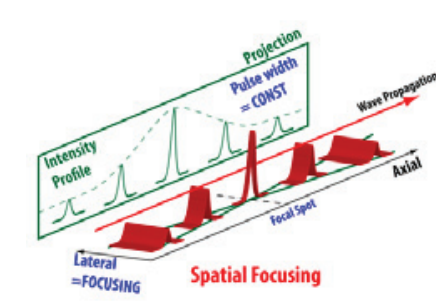

(a)

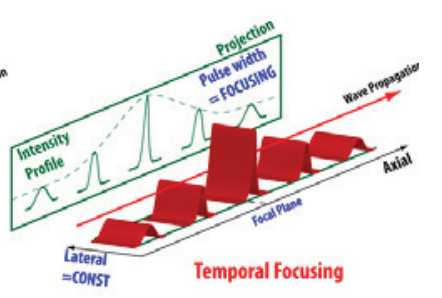

(b)
Fig. 1: Pictorial description of (a) spatial focusing and (b) temporal focusing

\section{Procedure, Results, \& Discussion}

By modeling DRWFI approach, we are able to optimize the excitation efficiency of this technique for both imaging and microfabrication. In terms of imaging, we have demonstrated that single quantum dots attached to integrin receptors in the cell membrane can be imaged (Fig. 2).

We have further demonstrated this imaging technique is compatible with spectroscopic analysis methods such as image correlation spectroscopy and single particle tracking. In terms of microfabrication, we have demonstrated 3D patterns can be written in 15 micron fluorescent microspheres via photobleaching.

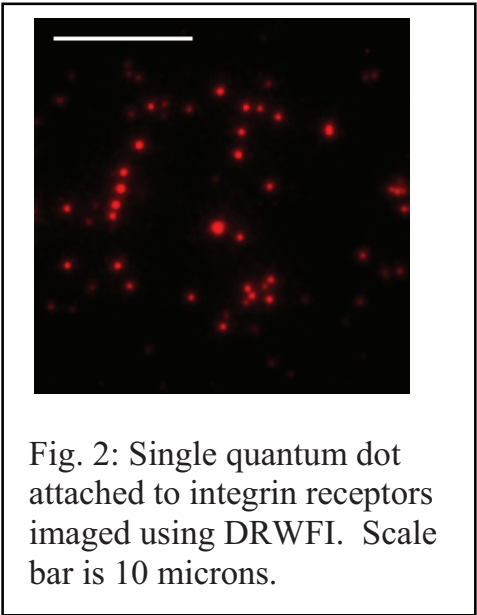

\section{CONCLUSION}

We have demonstrated single quantum dot imaging and 3D fabrication using temporal focusing based WFDRI. 


\section{ACKNOWLEDGMENT}

This work is support by Singapore-MIT Alliance and Singapore-MIT Alliance for Science \& Technology Center.

\section{REFERENCES}

[1] D. Oron, E. Tal, Y. Silberberg, "Scan-less depth resolved microscopy," Optics Express, vol 13, pp. 1468-1476, March 2005.

[2] G. H. Zhu, J. van Howe, M. Durst, R. W. Zipfel, C. Zu,

"Simultaneous spatial and temporal focusing of femtosecond pulses," Optics Express, vol.13, pp. 2153-2159, March 2005 